\title{
THE KAHN-PRIDDY THEOREM AND THE HOMOTOPY OF THE THREE-SPHERE
}

\author{
PIOTR BEBEN AND STEPHEN THERIAULT
}

(Communicated by Brooke Shipley)

\begin{abstract}
Let $p$ be an odd prime. The least nontrivial $p$-torsion homotopy group of $S^{3}$ occurs in dimension $2 p$ and is of order $p$. This induces a map $f: P^{2 p+1}(p) \rightarrow S^{3}$, where $P^{2 p+1}(p)$ is a mod- $p$ Moore space. An important conjecture related to the Kahn-Priddy Theorem is that the double loops on the three-connected cover of $f$ has a right homotopy inverse. We prove a weaker but still useful property: if $X$ is the cofiber of $f$, then the double loop on the three-connected cover of the inclusion $S^{3} \rightarrow X$ is null homotopic.
\end{abstract}

\section{INTRODUCTION}

Localize spaces and maps at an odd prime $p$, and take homology with mod- $p$ coefficients. For a space $X$, let $X\langle 3\rangle$ be the three-connected cover of $X$. For $m>1$ and $r \geq 1$, the mod- $p$ Moore space $P^{m}\left(p^{r}\right)$ is the homotopy cofiber of the degree $p^{r}$ map on $S^{m-1}$.

It is well known that the least nonvanishing torison homotopy group of $S^{3}$ occurs in dimension $2 p$ and has order $p$. Let $\alpha: S^{2 p} \longrightarrow S^{3}$ be a representative of this homotopy group. Since $\alpha$ has order $p$, it extends to a map $\bar{\alpha}: P^{2 p+1}(p) \longrightarrow S^{3}$. Taking three-connected covers, we obtain a map $\bar{\alpha}\langle 3\rangle: P^{2 p+1}(p) \longrightarrow S^{3}\langle 3\rangle$. Since $S^{3}\langle 3\rangle$ is the loops on $B S^{3}\langle 4\rangle, \bar{\alpha}\langle 3\rangle$ extends to a map $\widehat{\alpha}\langle 3\rangle: \Omega P^{2 p+2}(p) \longrightarrow S^{3}\langle 3\rangle$.

In [S1, Selick showed that $\Omega^{2} \widehat{\alpha}\langle 3\rangle$ has a right homotopy inverse. This is related to the Kahn-Priddy Theorem. Let $B\left(\mathbb{R}^{n}, p\right)$ be the configuration space of $p$-tuples of distinct points in $\mathbb{R}^{n}$. The Kahn-Priddy Theorem states that there is a map $\theta: \Omega^{\infty} \Sigma^{\infty} B\left(\mathbb{R}^{\infty}, p\right) \longrightarrow \Omega_{0}^{\infty} S^{\infty}$ which has a right homotopy inverse. Noting that $B\left(\mathbb{R}^{3}, p\right) \simeq P^{2 p-2}(p)$, Selick's result can be reformulated as in $[\mathrm{C}$ to show that there is a homotopy commutative diagram

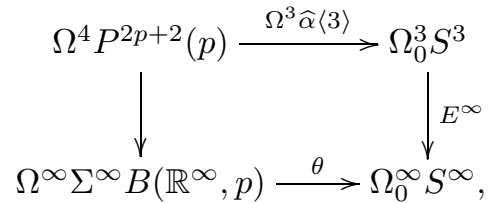

where $E^{\infty}$ is the stabilization map. Selick's right homotopy inverse for $\Omega^{3} \widehat{\alpha}\langle 3\rangle$ can therefore be regarded as a destabilization of the Kahn-Priddy Theorem for $S^{3}$.

Received by the editors July 1, 2011.

2010 Mathematics Subject Classification. Primary 55P35, 55Q40.

Key words and phrases. Three-sphere, homotopy group. 
It was conjectured by Cohen, Moore and Neisendorfer CMN2 that this destabilization can be "desuspended". Precisely, they conjecture that Selick's right homotopy inverse for $\Omega^{3} P^{2 p+2}(p) \stackrel{\Omega^{2} \widehat{\alpha}\langle 3\rangle}{\longrightarrow} \Omega^{2} S^{3}\langle 3\rangle$ can be improved to a right homotopy inverse for $\Omega^{2} P^{2 p+1}(p) \stackrel{\Omega^{2} \bar{\alpha}\langle 3\rangle}{\longrightarrow} \Omega^{2} S^{3}\langle 3\rangle$. Such an inverse would imply that the homotopy-theoretic properties of $S^{3}$ are governed by the Moore space $P^{2 p+1}(p)$. For example, suppose that $f: S^{3} \longrightarrow Y$ is a map such that the composite $P^{2 p+1}(p) \stackrel{\bar{\alpha}}{\longrightarrow} S^{3} \stackrel{f}{\longrightarrow} Y$ is null homotopic. Then the right homotopy inverse for $\Omega^{2} \bar{\alpha}$ implies that the map $\Omega^{2} S^{3}\langle 3\rangle \stackrel{\Omega^{2} f\langle 3\rangle}{\longrightarrow} \Omega^{2} Y\langle 3\rangle$ is null homotopic. In particular, $f$ induces the zero map on the homotopy groups $\pi_{m}$ for $m>3$.

At present, this conjecture seems out of reach with current techniques. However, it may be the case that some of its consequences can nevertheless be proved. In this paper we adopt that point of view and prove the following.

Theorem 1.1. For any space $Y$, let $f: S^{3} \longrightarrow Y$ be a map with the property that the composite $P^{2 p+1}(p) \stackrel{\bar{\alpha}}{\longrightarrow} S^{3} \stackrel{f}{\longrightarrow} Y$ is null homotopic. Then the map $\Omega^{2} S^{3}\langle 3\rangle \stackrel{\Omega^{2} f\langle 3\rangle}{\longrightarrow} \Omega^{2} Y\langle 3\rangle$ is null homotopic. Consequently, the induced map $\pi_{m}\left(S^{3}\right)$ $\stackrel{f_{*}}{\longrightarrow} \pi_{m}(Y)$ is zero for all $m>3$.

In the special case when $Y$ is an $H$-space, the authors proved a stronger result in [BT], that $\Omega f\langle 3\rangle$ is null homotopic. In this paper we use different methods to approach the general case when $Y$ is any space. Theorem 1.1 is a consequence of a universal example. Let $X$ be the homotopy cofiber of the map $P^{2 p+1}(p) \stackrel{\bar{\alpha}}{\longrightarrow} S^{3}$.

Theorem 1.2. The inclusion $S^{3} \longrightarrow X$ has the property that the map $\Omega^{2} S^{3}\langle 3\rangle \longrightarrow$ $\Omega^{2} X\langle 3\rangle$ is null homotopic.

Theorem 1.1 follows from Theorem 1.2 because a null homotopy for $f \circ \bar{\alpha}$ implies the existence of an extension of $S^{3} \stackrel{f}{\longrightarrow} Y$ to a map $X \longrightarrow Y$.

\section{SOME MOD- $p$ HOMOTOPY THEORY}

In this section we introduce the mod- $p$ homotopy theory necessary to prove the existence of a certain null homotopy, stated in Proposition 2.2 The modern reference for mod- $p$ homotopy theory is [N3. A space $Y$ is an $H$-group if it is a homotopy associative $H$-space with a homotopy inverse. For an $H$-group $Y$, let $c: Y \times Y \longrightarrow Y$ be the commutator, defined pointwise by $c(x, y)=x y x^{-1} y^{-1}$. Observe that $c$ restricts trivially to $Y \vee Y$, so it extends to a map

$$
\bar{c}: Y \wedge Y \longrightarrow Y \text {. }
$$

Since the connecting map for the cofibration $Y \vee Y \longrightarrow Y \times Y \rightarrow Y \wedge Y$ is null homotopic, the homotopy class of $\bar{c}$ is determined by that of $c$. Further, all of this is natural for $H$-maps $Y \longrightarrow Z$ between $H$-groups.

For any $s, t>1$, there is a homotopy equivalence $P^{s}(p) \wedge P^{t}(p) \simeq P^{s+t}(p) \vee$ $P^{s+t-1}(p)$. Since $p$ is an odd prime, there is a unique (up to homotopy) choice of inclusion $P^{s+t}(p) \longrightarrow P^{s}(p) \wedge P^{t}(p)$. Suppose that $Y$ is an $H$-group and there are maps $f: P^{s}(p) \longrightarrow Y$ and $g: P^{t}(p) \longrightarrow Y$. The mod- $p$ Samelson product of $f$ and $g$ is defined as the composite

$$
\langle f, g\rangle: P^{s+t}(p) \longrightarrow P^{s}(p) \wedge P^{t}(p) \stackrel{f \wedge g}{\longrightarrow} Y \wedge Y \stackrel{\bar{c}}{\longrightarrow} Y .
$$


We will now define iterated mod- $p$ Samelson products in a case of interest. Let $\nu: P^{2 n}(p) \longrightarrow \Omega P^{2 n+1}(p)$ be the adjoint of the identity map and $\mu: P^{2 n-1}(p) \longrightarrow$ $\Omega P^{2 n+1}(p)$ be the adjoint of the Bockstein map. Let $a d^{0}(\nu)=\mu$, and for $k \geq 1$ inductively define $a d^{k}(\nu)(\mu): P^{2 n(k+1)-1}(p) \longrightarrow \Omega P^{2 n+1}(p)$ as the mod- $p$ Samelson product $\left\langle\nu, a d^{k-1}(\nu)(\mu)\right\rangle$. We single out a special case: let

$$
\tau: P^{2 n p-1}(p) \longrightarrow \Omega P^{2 n+1}(p)
$$

be $a d^{p-1}(\nu)(\mu)$.

For $m>1$ and $r \geq 1$, let $\underline{p}^{r}: S^{m-1} \longrightarrow S^{m-1}$ be the map of degree $p^{r}$. Define maps $\omega$ and $\rho$ by the homotopy pushout

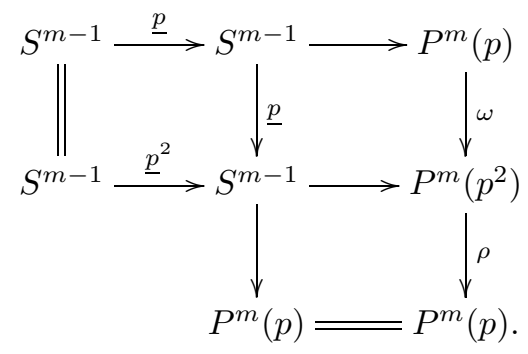

It is useful for later to note that the composite $P^{m}\left(p^{2}\right) \stackrel{\rho}{\longrightarrow} P^{m}(p) \stackrel{\omega}{\longrightarrow} P^{m}\left(p^{2}\right)$ is homotopic to the map of degree $p$. Let

$$
\delta: P^{m}(p) \longrightarrow P^{m+1}(p)
$$

be the connecting map for the cofibration in the right column.

As in [N3], the mod- $p$ Samelson product is bilinear, satisfies anti-commutativity and Jacobi identities, and the Bockstein acts on it as a derivation. These properties were used in [CMN1] to show that $\beta \tau$ is divisible by $p$, where $\beta$ is the Bockstein coming from the short exact sequence $0 \longrightarrow \mathbb{Z} \stackrel{p}{\longrightarrow} \mathbb{Z} \longrightarrow \mathbb{Z} / p \mathbb{Z} \longrightarrow 0$. This implies that the composite $P^{2 n p-2}(p) \stackrel{\delta}{\longrightarrow} P^{2 n p-1}(p) \stackrel{\tau}{\longrightarrow} \Omega P^{2 n+1}(p)$ is null homotopic, and therefore $\tau$ extends across $\omega$ to a map

$$
\bar{\tau}: P^{2 n p-1}\left(p^{2}\right) \longrightarrow \Omega P^{2 n+1}(p) .
$$

There may be many choices of such an extension. We first describe a choice of extension used in CMN2 . Let $S^{2 n+1}\{p\}$ be the fiber of the degree $p$ map on $S^{2 n+1}$ and let $i: P^{2 n+1}(p) \longrightarrow S^{2 n+1}\{p\}$ be the inclusion of the bottom Moore space. In CMN2 it was shown that the extension $\bar{\tau}$ can be chosen so that the composite $P^{2 n p-1}(p) \stackrel{\bar{\tau}}{\longrightarrow} \Omega P^{2 n+1}(p) \stackrel{\Omega i}{\longrightarrow} \Omega S^{2 n+1}\{p\}$ is null homotopic.

We wish to show that $\bar{\tau}$ can be chosen to satisfy a property that is stronger than $\Omega i \circ \bar{\tau} \simeq *$. A Serre spectral sequence calculation immediately shows that $H_{*}\left(S^{2 n+1}\{p\}\right) \cong H_{*}\left(\Omega S^{2 n+1}\right) \otimes H_{*}\left(S^{2 n+1}\right) \cong \mathbb{Z} / p \mathbb{Z}[x] \otimes \Lambda(y)$ for $|x|=2 n$ and $|y|=$ $2 n+1$. Let $M$ be the $(6 n+1)$-skeleton of $S^{2 n+1}\{p\}$, so $H_{*}(M) \cong\left\{x, y, x^{2}, x y, x^{3}\right.$, $\left.x^{2} y\right\}$. Note that the next element in $H_{*}\left(S^{2 n+1}\{p\}\right)$ is $x^{4}$, so $M$ can equally be regarded as the $(8 n-1)$-skeleton of $S^{2 n+1}\{p\}$. In Proposition 2.2 we improve on the result in CMN2 by showing that the extension $\bar{\tau}$ can be chosen so that the composite $P^{2 n p-1}(p) \stackrel{\bar{\tau}}{\longrightarrow} \Omega P^{2 n+1}(p) \longrightarrow \Omega M$ is null homotopic when $p \geq 5$. To explain how this occurs, we first consider relative Samelson products over an $H$-space, as presented in [N3, 6.11]. 
Since the degree $p$ map on $S^{2 n+1}$ is an $H$-map, its fibre $S^{2 n+1}\{p\}$ is an $H$-space. By [N1, the multiplication $\mu$ on $S^{2 n+1}\{p\}$ can be chosen so that it is stationary on the wedge and so that it is homotopy associative. To simplify notation, let $P^{m}=P^{m}(p)$. Given maps $a: X \longrightarrow S^{2 n+1}\{p\}$ and $b: Y \longrightarrow S^{2 n+1}\{p\}$, let $a \cdot b$ be the composite $X \times Y \stackrel{a \times b}{\longrightarrow} S^{2 n+1}\{p\} \times S^{2 n+1}\{p\} \stackrel{\mu}{\longrightarrow} S^{2 n+1}\{p\}$. Denote the wedge sum $X \vee Y \longrightarrow Z$ of two maps $c$ and $d$ by $c \perp d$. Observe that there are strictly commutative diagrams

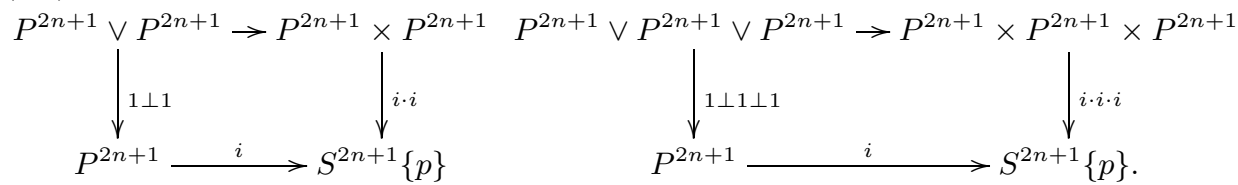

Note that the homotopy associativity of $S^{2 n+1}\{p\}$ ensures that the order of multiplication in the map $i \cdot i \cdot i$ is irrelevant.

Let $f: P^{s}(p) \longrightarrow \Omega P^{2 n+1}(p), g: P^{t}(p) \longrightarrow \Omega P^{2 n+1}(p)$ and $h: P^{q}(p) \longrightarrow$ $\Omega P^{2 n+1}(p)$ be maps. Let $f^{\prime}: \Sigma P^{s}(p) \longrightarrow P^{2 n+1}(p), g^{\prime}: \Sigma P^{t}(p) \longrightarrow P^{2 n+1}(p)$ and $h^{\prime}: \Sigma P^{q}(p) \longrightarrow P^{2 n+1}(p)$ be the adjoints of $f, g$ and $h$ respectively. By naturality, there are strictly commutative diagrams
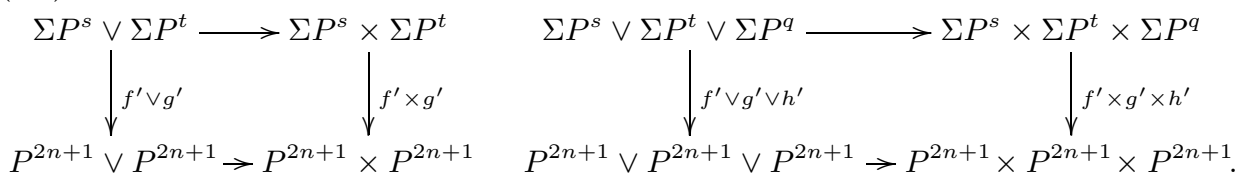

Juxtaposing the diagrams in (2.2) and (2.3) we obtain strictly commutative diagrams
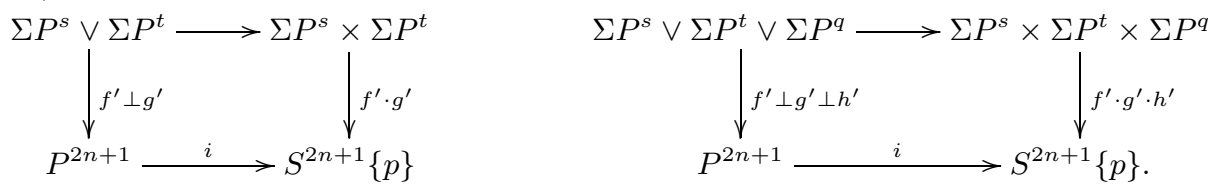

Next, let $N \longrightarrow \widetilde{P}^{2 n+1} \stackrel{\tilde{i}}{\longrightarrow} S^{2 n+1}\{p\}$ be the fibration which results from the standard replacement of $P^{2 n+1} \stackrel{i}{\longrightarrow} S^{2 n+1}\{p\}$. Note that this replacement comes with a homotopy equivalence $j: P^{2 n+1} \longrightarrow \widetilde{P}^{2 n+1}$ such that $\tilde{i} \circ j \simeq i$. Abusing notation, also use $f^{\prime}$ to denote the composite $\Sigma P^{m} \stackrel{f^{\prime}}{\longrightarrow} P^{2 n+1} \stackrel{j}{\longrightarrow} \widetilde{P}^{2 n+1}$. Then from (2.4) we obtain strictly commutative diagrams
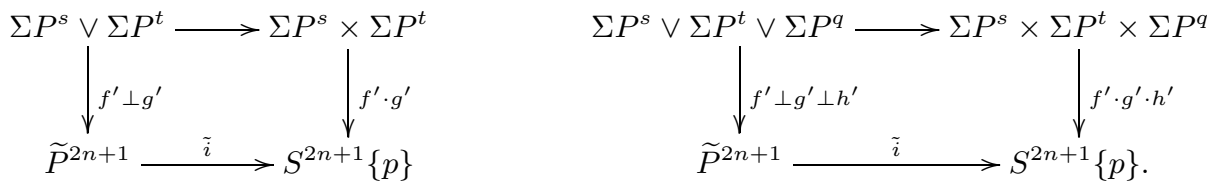

Let $F \longrightarrow \widetilde{P}^{s, t} \longrightarrow \Sigma P^{s} \times \Sigma P^{t}$ be the fibration which results from the standard replacement of $\Sigma P^{s} \vee \Sigma P^{t} \longrightarrow \Sigma P^{s} \times \Sigma P^{t}$. As in [N3, 6.11], there is an extension 
of $\Sigma P^{s} \vee \Sigma P^{t} \stackrel{f^{\prime} \perp g^{\prime}}{\longrightarrow} \widetilde{P}^{2 n+1}$ to a strictly commutative diagram

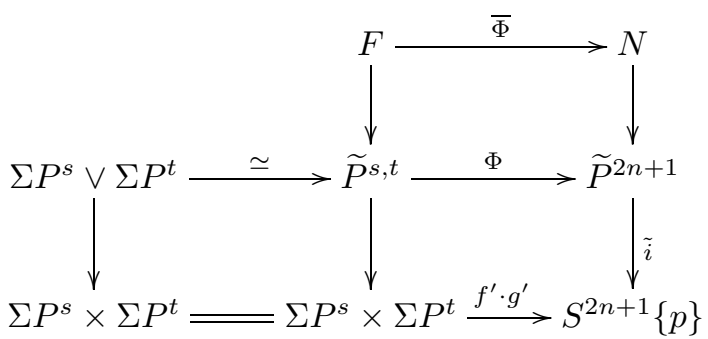

where the lift $\Phi$ is uniquely determined up to fibre homotopy, implying that the induced map of fibres $\bar{\Phi}$ is uniquely determined up homotopy.

By the Hilton-Milnor Theorem, the fibration $F \longrightarrow \widetilde{P}^{s, t} \longrightarrow \Sigma P^{s} \times \Sigma P^{t}$ splits after looping as $\Omega \widetilde{P}^{s, t} \simeq \Omega \Sigma P^{s} \times \Omega \Sigma P^{t} \times \Omega F$. Let $\iota_{s}: P^{s} \longrightarrow \Omega\left(\Sigma P^{s} \vee \Sigma P^{t}\right)$ and $\iota_{t}: P^{t} \longrightarrow \Omega\left(\Sigma P^{s} \vee \Sigma P^{t}\right)$ be the inclusions. The mod- $p$ Samelson product $P^{s+t} \stackrel{\left\langle\iota_{s}, \iota_{t}\right\rangle}{\longrightarrow} \Omega\left(\Sigma P^{s} \vee \Sigma P^{t}\right)$ maps to the basepoint in $\Omega \Sigma P^{s} \times \Omega \Sigma P^{t}$ and so lifts to a map

$$
\left\langle\iota_{s}, \iota_{t}\right\rangle_{r}: P^{s+t} \longrightarrow \Omega F \text {. }
$$

The splitting of $\Omega \widetilde{P}^{s, t}$ implies that the homotopy class of the lift is uniquely determined by that of $\left\langle\iota_{s}, \iota_{t}\right\rangle$, so $\left\langle\iota_{s}, \iota_{t}\right\rangle_{r}$ is well defined. The composite

$$
\langle f, g\rangle_{r}: P^{s+t} \stackrel{\left\langle\iota_{r}, \iota_{s}\right\rangle_{r}}{\longrightarrow} \Omega F \stackrel{\bar{\Phi}}{\longrightarrow} \Omega N
$$

is the relative Samelson product of $f$ and $g$. It is a choice of a lift of the mod-p Samelson product $\langle f, g\rangle$. In [N3, 6.11], it is shown that the relative Samelson product is bilinear, satisfies a twisted anti-commutativity property, and the Bockstein acts on it as a derivation.

One would also like the relative Samelson product to satisfy a Jacobi identity. To obtain this we turn to the left square in (2.5). Let $G \longrightarrow \widetilde{P}^{s, t, q} \longrightarrow \Sigma P^{s} \times \Sigma P^{t} \times \Sigma P^{q}$ be the fibration which results from the standard replacement of $\Sigma P^{s} \vee \Sigma P^{t} \vee \Sigma P^{s} \longrightarrow$ $\Sigma P^{s} \times \Sigma P^{t} \times \Sigma P^{q}$. As before, there is an extension of $\Sigma P^{s} \vee \Sigma P^{t} \vee \Sigma P^{q} \stackrel{f^{\prime} \perp g^{\prime} \perp h^{\prime}}{\longrightarrow}$ $\widetilde{P}^{2 n+1}$ to a strictly commutative diagram

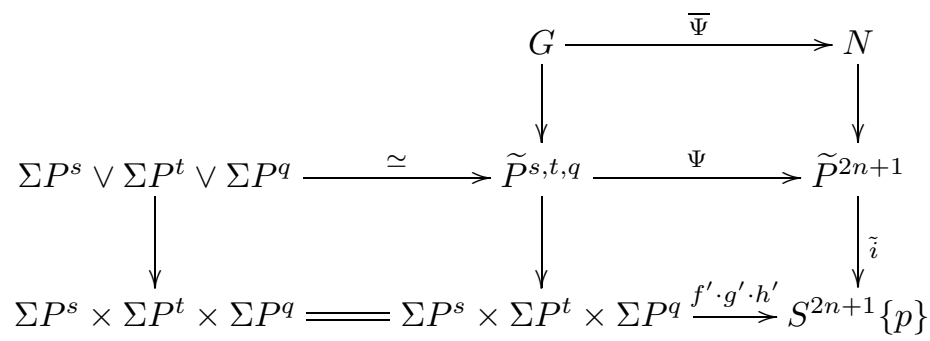

where the lift $\Psi$ is uniquely determined up to fibre homotopy, implying that the induced map of fibres $\bar{\Psi}$ is uniquely determined up to homotopy.

By the Hilton-Milnor Theorem, the fibration $G \longrightarrow \widetilde{P}^{s, t, q} \longrightarrow \Sigma P^{s} \times \Sigma P^{t} \times \Sigma P^{q}$ splits after looping as $\Omega \widetilde{P}^{s, t, q} \simeq \Omega \Sigma P^{s} \times \Omega \Sigma P^{t} \times \Omega \Sigma P^{q} \times \Omega G$. The mod- $p$ Samelson product $P^{s+t+q} \stackrel{\left\langle\iota_{s},\left\langle\iota_{t}, \iota_{q}\right\rangle\right\rangle}{\longrightarrow} \Omega P^{2 n+1}$ maps to the basepoint in $\Omega \Sigma P^{s} \times \Omega \Sigma P^{t} \times \Omega \Sigma P^{q}$ and so lifts to a map

$$
\left\langle\iota_{s}, \iota_{t}, \iota_{q}\right\rangle: P^{s+t+q} \longrightarrow \Omega G \text {. }
$$


The splitting of $\Omega \widetilde{P}^{s, t, q}$ implies that the homotopy class of the lift $\left\langle\iota_{s}, \iota_{t}, \iota_{q}\right\rangle$ is uniquely determined by that of $\left\langle\iota_{s},\left\langle\iota_{t}, \iota_{q}\right\rangle\right\rangle$, so $\left\langle\iota_{s}, \iota_{t}, \iota_{q}\right\rangle$ is well defined. Similarly, the mod- $p$ Samelson products $\left\langle\iota_{q},\left\langle\iota_{s}, \iota_{t}\right\rangle\right\rangle$ and $\left\langle\iota_{t},\left\langle\iota_{s}, \iota_{q}\right\rangle\right\rangle$ lift to well-defined maps

$$
\begin{gathered}
\left\langle\left\langle\iota_{s}, \iota_{t}, \iota_{q}\right\rangle\right\rangle: P^{s+t+q} \longrightarrow \Omega G, \\
\left\langle\left\langle\left\langle\iota_{s}, \iota_{t}, \iota_{q}\right\rangle\right\rangle\right\rangle: P^{s+t+q} \longrightarrow \Omega G,
\end{gathered}
$$

respectively. In [N3, 6.11] it is shown that $\left\langle\iota_{s}, \iota_{t}, \iota_{q}\right\rangle \simeq\left\langle\iota_{s},\left\langle\iota_{t}, \iota_{q}\right\rangle_{r}\right\rangle_{r},\left\langle\left\langle\iota_{q}, \iota_{s}, \iota_{t}\right\rangle\right\rangle \simeq$ $\left\langle\iota_{q},\left\langle\iota_{s}, \iota_{t}\right\rangle_{r}\right\rangle_{r},\left\langle\left\langle\left\langle\iota_{t}, \iota_{s}, \iota_{q}\right\rangle\right\rangle\right\rangle \simeq\left\langle\iota_{t},\left\langle\iota_{s}, \iota_{q}\right\rangle_{r}\right\rangle_{r}$, and if $p \geq 5$, there is a Jacobi identity

$$
\left\langle\iota_{s},\left\langle\iota_{t}, \iota_{q}\right\rangle_{r}\right\rangle_{r} \simeq(-1)^{q(s+t)+1}\left\langle\iota_{q},\left\langle\iota_{s}, \iota_{t}\right\rangle_{r}\right\rangle_{r}+(-1)^{s t}\left\langle\iota_{t},\left\langle\iota_{s}, \iota_{q}\right\rangle_{r}\right\rangle_{r} .
$$

Composing with $\bar{\Psi}$ then induces the Jacobi identity

$$
\left\langle f,\langle g, h\rangle_{r}\right\rangle_{r} \simeq(-1)^{q(s+t)+1}\left\langle h,\langle f, g\rangle_{r}\right\rangle_{r}+(-1)^{s t}\left\langle g,\langle f, h\rangle_{r}\right\rangle_{r} .
$$

Consequently, if $p \geq 5$, the relative Samelson product on $\Omega N$ is bilinear, satisfies anti-symmetry and Jacobi identities, and the Bockstein acts on it as a derivation.

Now we turn from $S^{2 n+1}\{p\}$ to its $(6 n+1)$-skeleton $M$ and reconsider relative Samelson products. We observed earlier that $M$ is also the $(8 n-1)$-skeleton of $S^{2 n+1}\{p\}$. So if $X$ is any $C W$-complex of dimension $\leq 8 n-2$, the skeletal inclusion $M \longrightarrow S^{2 n+1}\{p\}$ induces an isomorphism of sets $[X, M] \cong\left[X, S^{2 n+1}\{p\}\right]$. Abusing notation, if $X$ has dimension $\leq 8 n-2$ and there is a map $a: X \longrightarrow$ $S^{2 n+1}\{p\}$, we also write $a: X \longrightarrow M$ for its inverse image under the isomorphism $[X, M] \cong\left[X, S^{2 n+1}\{p\}\right]$. In our case, we obtain isomorphisms $\left[P^{2 n+1} \times P^{2 n+1}, M\right] \cong$ $\left[P^{2 n+1} \times P^{2 n+1}, S^{2 n+1}\{p\}\right]$ and $\left[P^{2 n+1} \times P^{2 n+1} \times P^{2 n+1}, M\right] \cong\left[P^{2 n+1} \times P^{2 n+1} \times\right.$ $\left.P^{2 n+1}, S^{2 n+1}\{p\}\right]$, so from (2.2) we obtain strictly commutative diagrams

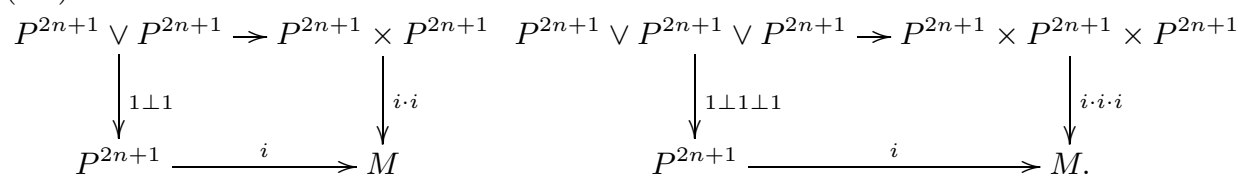

Juxtaposing with (2.3) we obtain strictly commutative diagrams
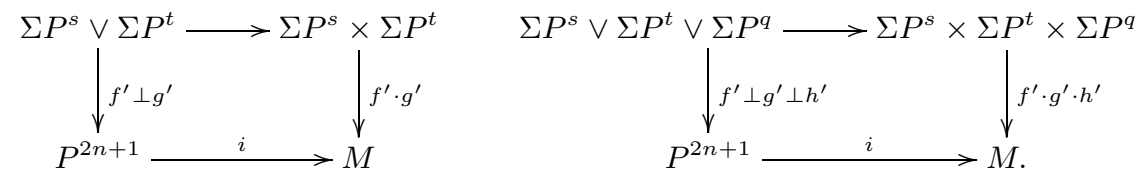

Let $\bar{N} \longrightarrow \bar{P}^{2 n+1} \stackrel{\bar{i}}{\longrightarrow} M$ be the fibration which results from the standard replacement of $P^{2 n+1} \stackrel{i}{\longrightarrow} M$. Now argue exactly as before with the fibration $\bar{N} \longrightarrow \bar{P}^{2 n+1} \stackrel{\bar{i}}{\longrightarrow} M$ in place of the fibration $N \longrightarrow \widetilde{P}^{2 n+1} \stackrel{\tilde{i}}{\longrightarrow} S^{2 n+1}\{p\}$ to define relative Samelson products $\langle,\rangle_{\bar{r}}$ on $\Omega \bar{N}$ and show that they satisfy bilinearity, anti-symmetry and Jacobi identities, and that the Bockstein acts as a derivation.

With these Lie identities in place, let $a d(\nu)(\mu)_{\bar{r}}=\langle\nu, \mu\rangle_{\bar{r}}$ and for $k>2$ inductively define $a d^{k}(\nu)(\mu)_{\bar{r}}=\left\langle\nu, a d^{k-1}(\nu)(\mu)_{\bar{r}}\right\rangle_{\bar{r}}$. Then the same argument as in CMN1 shows that the relative Samelson product $\tau_{\bar{r}}=a d^{p-1}(\nu)(\mu)_{\bar{r}}$ has the 
property that $\beta \tau_{\bar{r}}$ is divisible by $p$, implying that the composite $P^{2 n p-2}(p) \stackrel{\delta}{\longrightarrow}$ $P^{2 n p-1}(p) \stackrel{\tau_{\bar{r}}}{\longrightarrow} \Omega \bar{N}$ is null homotopic, and therefore the relative Samelson product $\tau_{\bar{r}}$ extends across $\omega$ to a map

$$
\bar{\tau}_{\bar{r}}: P^{2 n p-1}\left(p^{2}\right) \longrightarrow \Omega \bar{N} .
$$

Let $\bar{\tau}$ be the composite

$$
\bar{\tau}: P^{2 n p-1}\left(p^{2}\right) \stackrel{\bar{\tau}_{\bar{r}}}{\longrightarrow} \Omega \bar{N} \longrightarrow \Omega \bar{P}^{2 n+1} \stackrel{\simeq}{\longrightarrow} \Omega P^{2 n+1}(p) .
$$

By construction, $\bar{\tau}$ is an extension of the mod- $p$ Samelson product $\tau$. Summarizing, we have proved the following.

Lemma 2.1. If $p \geq 5$, the map $\bar{\tau}$ is a choice of an extension of the mod-p Samelson product $\tau$ with the property that the composite $P^{2 n p-1}(p) \stackrel{\bar{\tau}}{\longrightarrow} \Omega P^{2 n+1}(p) \longrightarrow \Omega M$ is null homotopic.

When $p=3$, the Jacobi identity for relative Samelson products may not hold, so we cannot use the same argument to produce a 3-primary extension of $\tau$ which composes trivially into $\Omega M$. However, in this case we can get around the lack of a Jacobi identity by using a low-dimensional argument. Consider again the extension $P^{6 n-1}\left(3^{2}\right) \stackrel{\bar{\tau}}{\longrightarrow} \Omega P^{2 n+1}(3)$ of $\tau$ in CMN2 which has the property that the composition $P^{6 n-1}\left(3^{2}\right) \stackrel{\bar{\tau}}{\longrightarrow} \Omega P^{2 n+1}(3) \longrightarrow \Omega S^{2 n+1}\{3\}$ is null homotopic. By definition, $M$ is the $(6 n+1)$-skeleton of $S^{2 n+1}\{3\}$. Thus for any space $X$ of dimension less than $6 n$, the looped inclusion $\Omega M \longrightarrow \Omega S^{2 n+1}\{3\}$ induces an isomorphism $[X, \Omega M] \cong$ $\left[X, \Omega S^{2 n+1}\{3\}\right]$. This implies that the composite $P^{6 n-1}\left(3^{2}\right) \stackrel{\bar{\tau}}{\longrightarrow} P^{2 n+1}(3) \longrightarrow \Omega M$ is null homotopic, and therefore we obtain the 3-primary analogue of Lemma 2.1 .

Now for $p \geq 3$, let $t: P^{2 n p}(p) \longrightarrow P^{2 n+1}(p)$ be the adjoint of $\tau$, and let $\bar{t}: P^{2 n p}\left(p^{3}\right) \longrightarrow P^{2 n+1}(p)$ be the adjoint of $\bar{\tau}$. Then Lemma 2.1 for $p \geq 5$ and the preceding paragraph for $p=3$ imply the following.

Proposition 2.2. For $p \geq 3$, the map $\bar{t}$ is a choice of extension of the mod-p Whitehead product $t$ with the property that the composite $P^{2 n p}\left(p^{2}\right) \stackrel{\bar{t}}{\longrightarrow} P^{2 n+1}(p) \longrightarrow M$ is null homotopic.

\section{A LIFT}

In this section we prove the existence of a certain lift in Lemma 3.5. For $m \geq 3$, let $\underline{p}: P^{m}\left(p^{2}\right) \longrightarrow P^{m}\left(p^{2}\right)$ be the map of degree $p$, and let $C^{m}$ be its homotopy cofiber. (In fact, $C^{m} \simeq P^{m}(p) \vee P^{m+1}(p)$, but we will not need this.) Define spaces $G_{1}$ and $\bar{G}_{1}$ by the homotopy pushout

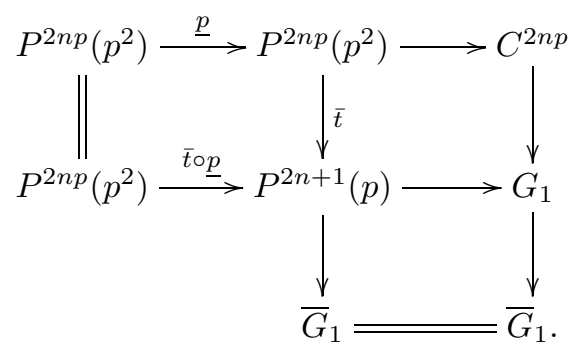


Observe that there is also a homotopy pushout diagram

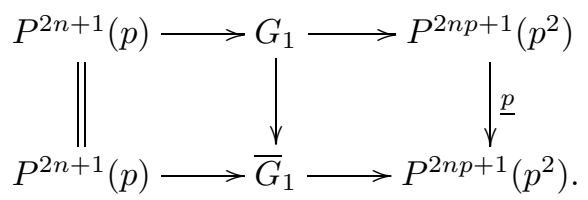

The definition of $\bar{G}_{1}$ as the cofiber of $\bar{t}$ and the null homotopy for the composition $P^{2 n p}\left(p^{2}\right) \stackrel{\bar{t}}{\longrightarrow} P^{2 n+1}(p) \longrightarrow M$ in Proposition 2.2 immediately imply the following.

Lemma 3.1. There is a homotopy commutative diagram

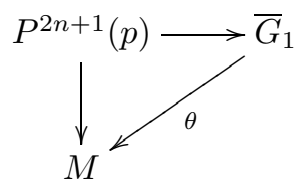

for some map $\theta$.

Let $\varpi$ be the composite

$$
\varpi: G_{1} \longrightarrow \bar{G}_{1} \stackrel{\theta}{\longrightarrow} M \longrightarrow S^{2 n+1}\{p\} .
$$

Since $G_{1} \longrightarrow \bar{G}_{1}$ is the identity on the bottom Moore space $P^{2 n+1}(p), \theta$ is an extension of the inclusion $P^{2 n+1}(p) \longrightarrow M$, and $M$ is the $(6 n+1)$-skeleton of $S^{2 n+1}\{p\}$, we obtain the following.

Lemma 3.2. There is a homotopy commutative diagram

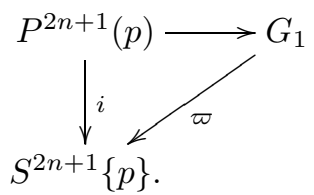

That is, Lemma 3.2 gives an explicit choice of an extension of the inclusion $i$ to $G_{1}$.

Now specialize to the case of primary interest, when $n=p$. We wish to relate $G_{1} \stackrel{\varpi}{\longrightarrow} S^{2 p+1}\{p\}$ to the map $P^{2 p+1}(p) \stackrel{\bar{\alpha}}{\longrightarrow} S^{3}$. Recall that $\bar{\alpha}$ is an extension of the map $S^{2 p} \stackrel{\alpha}{\longrightarrow} S^{3}$ representing the least nontrivial $p$-torsion homotopy group of $S^{3}$. Since $S^{3}$ has a classifying space $B S^{3}$, the map $\alpha$ has an adjoint $a: S^{2 p+1} \longrightarrow B S^{3}$. Let $B$ be the homotopy fiber of $a$. Since $\alpha$ has order $p$, so does $a$. This implies that the degree $p$ map $S^{2 p+1} \stackrel{p}{\longrightarrow} S^{2 p+1}$ lifts to $B$. From this lift we obtain a homotopy fibration diagram

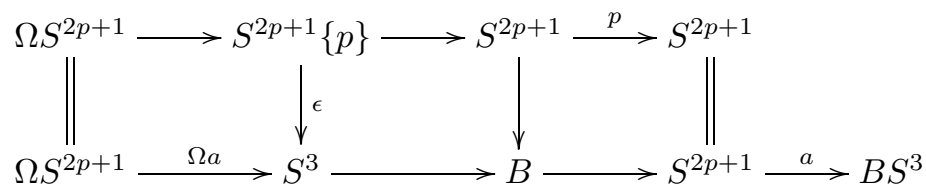

which defines the map $\epsilon$. Let $\bar{a}$ be the composite

$$
\bar{a}: P^{2 p+1}(p) \stackrel{i}{\longrightarrow} S^{2 p+1}\{p\} \stackrel{\epsilon}{\longrightarrow} S^{3} .
$$


Lemma 3.3. The map $\bar{a}$ is an extension of $\alpha$, and $\bar{a}$ is homotopic to $\bar{\alpha}$.

Proof. Let $E: S^{2 n} \longrightarrow \Omega S^{2 n+1}$ be the suspension. The definition of $a$ as the adjoint of $\alpha$ implies that $\Omega a \circ E \simeq \alpha$. The homotopy commutativity of the leftmost square in (3.1) then implies that the composite $S^{2 p} \stackrel{E}{\longrightarrow} \Omega S^{2 p+1} \longrightarrow S^{2 p+1}\{p\} \stackrel{\epsilon}{\longrightarrow} S^{3}$ is homotopic to $\alpha$. But by connectivity, this is homotopic to the composite $S^{2 p} \hookrightarrow$ $P^{2 p+1}(p) \stackrel{i}{\longrightarrow} S^{2 p+1}\{p\} \stackrel{\epsilon}{\longrightarrow} S^{3}$. Therefore, as $\bar{a}=\epsilon \circ i$ by definition, $\bar{a}$ is an extension of $\alpha$.

To show that $\bar{a}$ is homotopic to $\bar{\alpha}$, we show that any two extensions $a_{1}$ and $a_{2}$ of $\alpha$ are homotopic. Since both $a_{1}$ and $a_{2}$ extend $\alpha$, the difference $a_{1}-a_{2}$ factors through a map $S^{2 p+1} \longrightarrow S^{3}$. But $\pi_{2 p+1}\left(S^{3}\right)=0$. Hence $a_{1} \simeq a_{2}$.

Combining Lemmas 3.2 and 3.3 , we obtain the following.

Lemma 3.4. There is a homotopy commutative diagram

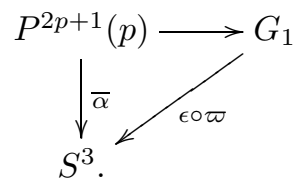

Recall that $X$ is the homotopy cofiber of the map $\bar{\alpha}$. Let $F$ be the homotopy fiber of the inclusion $S^{3} \longrightarrow X$. Observe that $P^{2 p+1}(p) \stackrel{\bar{\alpha}}{\longrightarrow} S^{3}$ lifts to $F$. In Proposition 3.5 we prove a stronger statement, that $G_{1} \stackrel{\epsilon \circ \varpi}{\longrightarrow} S^{3}$ lifts to $F$.

Proposition 3.5. There is a lift

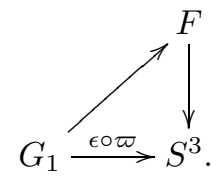

Proof. By definition of $\varpi, \epsilon \circ \varpi$ is the composite $G_{1} \longrightarrow \bar{G}_{1} \stackrel{\theta}{\longrightarrow} M \longrightarrow S^{2 p+1}\{p\}$ $\stackrel{\epsilon}{\longrightarrow} S^{3}$. By Lemmas 3.2 and 3.3 , the first three maps in this sequence are the identity on the bottom Moore space while $\epsilon$ restricted to the bottom Moore space is $\bar{\alpha}$. This implies that there is an iterated homotopy pushout diagram

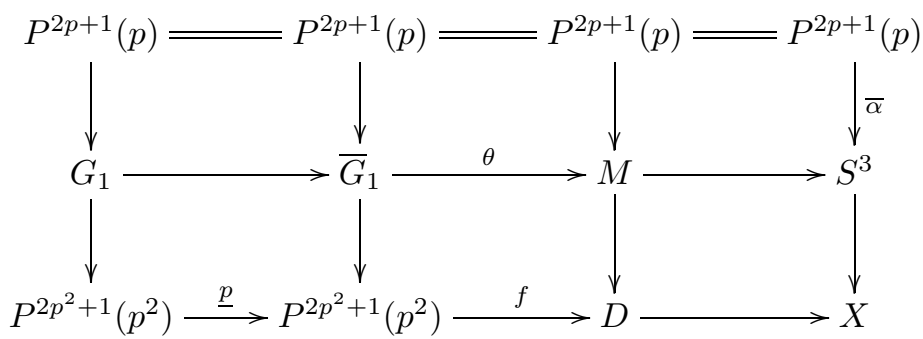

which defines the space $D$ and the map $f$.

We claim that $D \simeq P^{4 p+1}(p) \vee P^{6 p+1}(p)$. By CMN1, there is a homotopy equivalence $\Sigma S^{2 n+1}\{p\} \simeq \bigvee_{k=1}^{\infty} \Sigma P^{2 n k+1}(p)$. Since $M$ is the $(6 n+1)$-skeleton of $S^{2 n+1}\{p\}$, we then have $\Sigma M \simeq \bigvee_{k=1}^{3} \Sigma P^{2 n k+1}(p)$. Consequently, $\Sigma D \simeq \Sigma P^{4 n+1}(p) \vee$ $\Sigma P^{6 n+1}(p)$. The Hilton-Milnor Theorem therefore implies that the $(8 n-1)$-skeleton 
of $\Omega \Sigma D$ is homotopy equivalent to $P^{4 n+1}(p) \vee P^{6 n+1}(p)$. On the other hand, the Bott-Samelson Theorem states that $H_{*}(\Omega \Sigma D) \cong T\left(\widetilde{H}_{*}(D)\right)$, and the suspension $D \stackrel{E}{\longrightarrow} \Omega \Sigma D$ induces the inclusion of the generating set in homology. Since $D$ is $(4 n-1)$-connected, $H_{*}(\Omega \Sigma D)$ only has elements of tensor length one in degress less than $8 n$. That is, $E_{*}$ is an isomorphism in degrees $\leq 8 n-1$. Thus the composite $D \stackrel{E}{\longrightarrow}(\Omega \Sigma D)_{8 n-1} \stackrel{\simeq}{\longrightarrow} P^{4 n+1}(p) \vee P^{6 n+1}(p)$ is an isomorphism in homology, and so is a homotopy equivalence.

We now use an exponent argument to show that the map $f$ has order $p$. It is equivalent to showing that the double adjoint

$$
f^{\prime}: P^{2 p^{2}-2}\left(p^{2}\right) \longrightarrow \Omega^{2}\left(P^{4 p+1}(p) \vee P^{6 p+1}(p)\right)
$$

of $f$ has order $p$. The Hilton-Milnor Theorem and the fact that $P^{s}(p) \wedge P^{t}(p) \simeq$ $P^{s+t}(p) \vee P^{s+t-1}(p)$ implies that there is a homotopy equivalence

$$
\Omega\left(P^{4 p+1}(p) \vee P^{6 p+1}(p)\right) \simeq \prod_{\gamma \in \mathcal{I}} \Omega P^{m(\gamma)}(p)
$$

for some index set $\mathcal{I}$, where each $P^{m(\gamma)}(p)$ has dimension at least $4 p+1$. Upon looping, the $p^{t h}$-power map on $\Omega^{2}\left(P^{4 p+1}(p) \vee P^{6 p+1}(p)\right)$ is homotopic to the product of the $p^{t h}$-power maps on the factors $\Omega^{2} P^{m(\gamma)}(p)$. By [N1], the $p^{t h}$-power map on $\Omega^{2} P^{2 n+1}(p)$ is null homotopic in dimensions less than $2 n p-3$ and the $p^{\text {th }}$-power map on $\Omega^{2} P^{2 n+2}(p)$ is null homotopic in dimensions less than $4 n p-3$. Thus the $p^{t h}$-power map on $\Omega^{2}\left(P^{4 p+1}(p) \vee P^{6 p+1}(p)\right)$ is null homotopic in dimensions less than $4 p^{2}-3$. Hence $f^{\prime}$ has order $p$.

Since $f$ has order $p$, the composite $f \circ p$ in (3.2) is null homotopic. Therefore the homotopy commutativity of (3.2) implies that the composite $G_{1} \longrightarrow \bar{G}_{1} \stackrel{\theta}{\longrightarrow}$ $M \longrightarrow S^{2 p+1}\{p\} \stackrel{\epsilon}{\longrightarrow} S^{3} \longrightarrow X$ is null homotopic. That is, the composite $G_{1} \stackrel{\varpi}{\longrightarrow}$ $S^{2 p+1}\{p\} \stackrel{\epsilon}{\longrightarrow} S^{3} \longrightarrow X$ is null homotopic, so $\epsilon \circ \varpi$ lifts to $F$, as asserted.

\section{The PRoof of TheOrem 1.2}

We begin by introducing some background regarding Anick's fibration and related spaces. As motivation, recall that the Bott-Samelson Theorem states that there is an algebra isomorphism $H_{*}\left(\Omega P^{2 n+1}(p)\right) \cong T\left(\widetilde{H}_{*}\left(P^{2 n}(p)\right)\right) \cong T(u, v)$, where homology is taken with mod- $p$ coefficients, $T()$ is the free tensor algebra functor, $|v|=2 n$ and $u=\beta v$. Abelianizing, we obtain a map $T(u, v) \longrightarrow S(u, v)$, where $S()$ is the free symmetric algebra functor. The algebra $S(u, v)$ and the abelianization map can be realized geometrically. Parts (a) and (b) of the following theorem were originally proved in [A] for $p \geq 5$ and part (c) was proved in [S2]; a vastly simpler proof of parts (a) and (b) was later given in [GT], which is also valid for $p=3$.

Theorem 4.1. There is a homotopy fibration $S^{2 n-1} \longrightarrow T^{2 n+1}(p) \longrightarrow \Omega S^{2 n+1}$ satisfying:

(a) $H_{*}\left(T^{2 n+1}(p)\right) \cong S(u, v)$;

(b) there is a map $\Omega P^{2 n+1}(p) \longrightarrow T^{2 n+1}(p)$ with the property that

it induces the abelianization map $T(u, v) \longrightarrow S(u, v)$ in homology;

(c) there is a homotopy equivalence $T^{2 p+1}(p) \simeq \Omega S^{3}\langle 3\rangle$.

The space $G_{1}$ from Section 3 arises in the construction of the space $T^{2 n+1}(p)$. To be clear, we defined $G_{1}$ as the homotopy cofiber of $P^{2 n p}\left(p^{2}\right) \stackrel{\bar{t} \circ p}{\longrightarrow} P^{2 n+1}(p)$, 
where $\bar{t}$ is a particular choice of an extension for the adjoint of the mod- $p$ Samelson product $\tau$. In $\mathrm{AG}$ or $\mathrm{GT}$, the space labelled there as $G_{1}$ is the homotopy cofiber of a map $P^{2 n p}\left(p^{2}\right) \stackrel{\hat{t} \circ p}{\longrightarrow} P^{2 n+1}(p)$, where $\hat{t}$ is a possibly different choice of an extension for the adjoint of the mod- $p$ Samelson product $\tau$. However, recall from (2.1) that the degree $p$ map on $P^{2 n p}\left(p^{2}\right)$ is homotopic to the composite $P^{2 n p}\left(p^{2}\right) \stackrel{\rho}{\longrightarrow} P^{2 n p}(p) \stackrel{\omega}{\longrightarrow}$ $P^{2 n p}\left(p^{2}\right)$. As $\bar{t}$ and $\hat{t}$ both extend the adjoint $t$ of the mod-p Samelson product $\tau$, we have $\bar{t} \circ \omega \simeq t \simeq \hat{t} \circ \omega$. Thus $\bar{t} \circ p \simeq \hat{t} \circ p$. Therefore the space we label as $G_{1}$ and the space that $\left[\mathrm{AG}\right.$. or $\left[\mathrm{GT}\right.$ ] labels as $\overline{G_{1}}$ are identical. So the following result from [AG] or [GT holds.

Theorem 4.2. The map $\Omega P^{2 n+1}(p) \longrightarrow T^{2 n+1}(p)$ in Theorem 4.1 factors as a composite $\Omega P^{2 n+1}(p) \longrightarrow \Omega G_{1} \longrightarrow T^{2 n+1}(p)$.

In addition, the following theorem was proved for $p \geq 5$ following Anick's work. In light of the subsequent work in GT], inspection of the proof shows that it is also valid for $p=3$.

Theorem 4.3. There is a map $\Omega T^{2 n+1}(p) \longrightarrow \Omega^{2} G_{1}$ which is a right homotopy inverse of the loop map $\Omega^{2} G_{1} \longrightarrow \Omega T^{2 n+1}(p)$.

Combining this material on Anick's fibration with Proposition 3.5 we prove Theorem 1.2 .

Proof of Theorem 1.2. Let $j: S^{3} \longrightarrow X$ be the inclusion of the bottom cell. The lift of $G_{1} \stackrel{\epsilon \circ \varpi}{\longrightarrow} S^{3}$ to the fiber $F$ of $j$ in Proposition 3.5] implies that the composite $G_{1} \stackrel{\epsilon \circ \varpi}{\longrightarrow} S^{3} \stackrel{j}{\longrightarrow} X$ is null homotopic. Taking three-connected covers, the composite $G_{1} \stackrel{(\epsilon \circ \varpi)\langle 3\rangle}{\longrightarrow} S^{3}\langle 3\rangle \stackrel{j\langle 3\rangle}{\longrightarrow} X\langle 3\rangle$ is null homotopic. Note that $S^{3}\langle 3\rangle=P^{2 p+1}(p) \cup$ higher, and the three-connected cover of the map $P^{2 p+1}(p) \stackrel{\bar{\alpha}}{\longrightarrow} S^{3}$ is the inclusion of the bottom Moore space into $S^{3}\langle 3\rangle$. By Lemma 3.4 $\epsilon \circ \varpi$ is an extension of $\bar{\alpha}$, so the composition $P^{2 p+1}(p) \longrightarrow G_{1} \stackrel{(\epsilon \circ \varpi)\langle 3\rangle}{\longrightarrow} S^{3}\langle 3\rangle$ is the inclusion of the bottom Moore space. In particular, $(\epsilon \circ \varpi)\langle 3\rangle$ is degree one on the bottom cell.

By Theorems 4.1(b) and 4.2, there is a map $r: \Omega G_{1} \longrightarrow T^{2 p+1}(p)$ with the property that the composite $\Omega P^{2 n+1}(p) \longrightarrow \Omega G_{1} \stackrel{r}{\longrightarrow} T^{2 p+1}(p)$ induces the abelianization map in homology. In particular, $r$ is degree one on the bottom cell, and therefore so is $\Omega r$. By Theorem 4.3, there is a map $s: \Omega T^{2 p+1}(p) \longrightarrow \Omega^{2} G_{1}$ which is a left homotopy inverse of $\Omega r$. Thus $s$ is also degree one on the bottom cell.

To simplify notation, let $\phi=(\epsilon \circ \varpi)\langle 3\rangle$. Consider the composite

$$
\Omega T^{2 p+1}(p) \stackrel{s}{\longrightarrow} \Omega^{2} G_{1} \stackrel{\Omega^{2} \phi}{\longrightarrow} \Omega^{2} S^{3}\langle 3\rangle \stackrel{\Omega^{2} j\langle 3\rangle}{\longrightarrow} \Omega^{2} X\langle 3\rangle .
$$

By Theorem 4.1 (c), there is a homotopy equivalence $e: \Omega T^{2 p+1}(p) \stackrel{\simeq}{\longrightarrow} \Omega^{2} S^{3}\langle 3\rangle$. Since $s$ and $\Omega^{2} \phi$ are both degree one on the bottom Moore space, the composite $\Omega^{2} \phi \circ s \circ e^{-1}$ is a self-map of $\Omega^{2} S^{3}\langle 3\rangle$ which is degree one on the bottom cell. By [CM], any self-map of $\Omega^{2} S^{3}\langle 3\rangle$ which is degree one on the bottom cell is a homotopy equivalence. Thus $\Omega^{2} \phi \circ s \circ e^{-1}$ is a homotopy equivalence, which implies that $\Omega^{2} \phi \circ s$ is a homotopy equivalence. On the other hand, by the first paragraph of the proof, $j\langle 3\rangle \circ \phi$ is null homotopic. Thus $\Omega^{2} j\langle 3\rangle \circ \Omega^{2} \phi \circ s$ is null homotopic. Since $\Omega^{2} \phi \circ s$ is a homotopy equivalence, we therefore have that $\Omega^{2} j\langle 3\rangle$ is null homotopic, as asserted. 


\section{A stable generalization}

In this section we consider suspensions of the inclusion $S^{3} \stackrel{j}{\longrightarrow} X$ and how they behave on homotopy groups. Since $X$ is the universal example for the spaces $Y$ considered in Theorem [1.1, the results can be phrased in terms of any map $S^{3} \longrightarrow Y$.

By CMN2, the $p$-torsion in the homotopy groups of $S^{2 n+1}$ is annihilated by $p^{n}$. Thus if $Y$ is any space and $f: S^{2 n+1} \longrightarrow Y$ is any map, then $p^{n} \cdot \pi_{m}(f)=0$ for any $m>2 n+1$. In the case of $S^{3}$ when $n=1$, we obtain $p \cdot \pi_{m}(f)=0$ for $m>3$. Theorem 1.1 says that if $Y$ has the additional property that $f \circ \bar{\alpha}$ is null homotopic, then we have the stronger property that $\pi_{m}(f)=0$ for $m>3$. We generalize this as follows.

Proposition 5.1. Let $Y$ be a space and suppose there is a map $f: S^{3} \longrightarrow Y$ with the property that the composite $P^{2 p+1}(p) \stackrel{\bar{\alpha}}{\longrightarrow} S^{3} \stackrel{f}{\longrightarrow} Y$ is null homotopic. Then the map $S^{2 n+1} \stackrel{\Sigma^{2 n-2} f}{\longrightarrow} \Sigma^{2 n-2} Y$ has the property that $p^{n-1} \cdot \pi_{m}(f)=0$ for $m>2 n+1$.

Proof. As with Theorem 1.1, the proposition follows as a consequence of it's holding for the universal example $S^{3} \stackrel{j}{\longrightarrow} X$.

By CMN2, the $p^{t h}$-power map on $\Omega^{2} S^{2 n+1}$ factors as a composite $\Omega^{2} S^{2 n+1} \stackrel{\phi}{\longrightarrow}$ $S^{2 n-1} \stackrel{E^{2}}{\longrightarrow} \Omega^{2} S^{2 n+1}$ for some map $\phi$, where $E^{2}$ is the double suspension. Iterating we obtain a factorization of the $p^{n-1}$-power map on $\Omega^{2 n-2} S^{2 n+1}$ as a composite $\Omega^{2 n-2} S^{2 n+1} \stackrel{\varphi}{\longrightarrow} S^{3} \stackrel{E^{2 n-2}}{\longrightarrow} \Omega^{2 n-2} S^{2 n+1}$, where $E^{2 n-2}$ is the $(2 n-2)$-suspension map. The naturality of $E^{2 n-2}$ implies that there is a homotopy commutative diagram

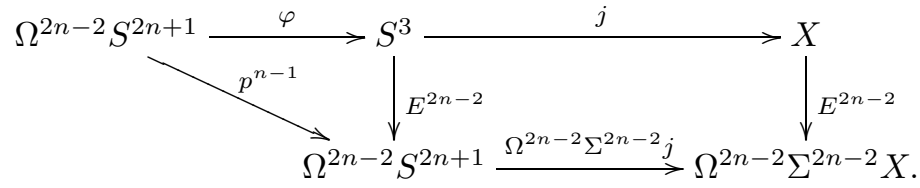

By Theorem 1.2, $\pi_{m}(j)=0$ for $m>3$. Thus the commutativity of the diagram implies that $p^{n-1} \cdot \pi_{m}\left(\Omega^{2 n-2} \Sigma^{2 n-2} j\right)=0$ for $m>3$. Equivalently, $p^{n-1} \cdot \pi_{m}\left(\Sigma^{2 n-2} j\right)=$ 0 for $m>2 n+1$.

\section{REFERENCES}

[A] D. Anick, Differential algebras in topology, Research Notes in Math. 3, A K Peters, 1993. MR $1213682(94 \mathrm{~h}: 55020)$

[AG] D. Anick and B. Gray, Small $H$-spaces related to Moore spaces, Topology 34 (1995), 859-881. MR 1362790 (97a:55011)

[BT] P. Beben and S. Theriault, Torsion in finite $H$-spaces and the homotopy of the threesphere, Homology, Homotopy Appl. 12 (2010), 25-37. MR 2721030

[C] F.R. Cohen, Two-primary analogues of Selick's theorem and the Kahn-Priddy theorem for the 3-sphere, Topology 23 (1984), 401-421. MR780733 (86e:55020)

[CM] F. Cohen and M. Mahowald, A remark on the self-maps of $\Omega^{2} S^{2 n+1}$, Indiana Univ. Math. J. 30 (1981), 583-588. MR620268(82i:55013)

[CMN1] F.R. Cohen, J.C. Moore and J.A. Neisendorfer, Torsion in homotopy groups, Ann. of Math. (2) 109 (1979), 121-168. MR.519355 (80e:55024)

[CMN2] F.R. Cohen, J.C. Moore and J.A. Neisendorfer, The double suspension and exponents of the homotopy groups of spheres, Ann. of Math. (2) 110 (1979), 549-565. MR.554384 (81c:55021) 
[G] B. Gray, On the iterated suspension, Topology 27 (1988), 301-310. MR963632 (89h:55016)

[GT] B. Gray and S. Theriault, An elementary construction of Anick's fibration, Geom. Topol. 14 (2010), 243-275. MR2578305 (2011a:55013)

[J] I.M. James, Reduced product spaces, Ann. of Math. (2) 62 (1955), 170-197. MR.0073181 $(17: 396 \mathrm{~b})$

[KP] D.S. Kahn and S.B. Priddy, The transfer and stable homotopy theory, Math. Proc. Camb. Philos. Soc. 83 (1978), 103-111. MR0464230(57:4164b)

[N1] J.A. Neisendorfer, Properties of certain H-spaces, Quart. J. Math. Oxford 34 (1983), 201-209. MR698206 (84h:55007)

[N2] J.A. Neisendorfer, The exponent of a Moore space, Algebraic Topology and Algebraic Ktheory (Princeton, NJ, 1983), 72-100, Ann. of Math. Study 113, Princeton University Press, 1987. MR.921472(89e:55029)

[N3] J.A. Neisendorfer, Algebraic methods in unstable homotopy theory, New Mathematical Monographs, vol. 12, Cambridge University Press, Cambridge, 2010. MR 2604913 (2011j:55026)

[S1] P.S. Selick, Odd primary torsion in $\pi_{k}\left(S^{3}\right)$, Topology 17 (1978), 407-412. MR.516219 (80c:55010)

[S2] P.S. Selick, Space exponents for loop spaces of spheres, Stable and unstable homotopy theory (Toronto, ON, 1996), 279-283, Fields Inst. Commun. 19, Amer. Math. Soc., 1998. MR 1622353 (99b:55013)

[T] S.D. Theriault, Anick's space and the double loops on odd primary Moore spaces, Trans. Amer. Math. Soc. 353 (2001), 1551-1566. MR.1709779 (2001f:55011)

Institute of Mathematics, Academy of Mathematics and Systems Science, Chinese Academy of Sciences, Beijing 100190, People's Republic of China

E-mail address: bebenp@unbc.ca

Department of Mathematical Sciences, University of Aberdeen, Aberdeen AB24 3 UE, UNITED KINGDOM

E-mail address: s.theriault@abdn.ac.uk 